\title{
Developing a spatio-temporal model of risk management for earthquake life detection rescue team
}

\author{
${ }^{1 *}$ A. R. Vafaeinezhad; ${ }^{1}$ A. A. Alesheikh; ${ }^{2}$ J. Nouri \\ ${ }^{1}$ Department of GIS Engineering, K.N. Toosi University of Technology, Tehran, Iran \\ ${ }^{2}$ Department of Environmental Management, Graduate School of the Environment and Energy, \\ Science and Research Branch, Islamic Azad University, Tehran, Iran
}

Received 18 January 2010; $\quad$ revised 5 February 2010; accepted 22 February 2010; avaiable online 1 March 2010

\begin{abstract}
Crisis management is a time-critical and collaborative activity that requires rapid assessment and decision- making. This study aims to develop a framework to increase teamwork efficiency by spatio-temporal modeling of human group activities in geospatial information systems. For this purpose, time geography framework was extended with round-robin scheduling and then was integrated in geographic information system environment to manage human activities in a spatio-temporal framework. The proposed framework enjoys the graphical presentation of the tasks to be executed, as well as mathematical modeling of the complex interactions to be managed. To validate the proposed model, activities of an earthquake life-detection team in normal and suggested framework were simulated and assessed. An average improvement of $18.69 \%$ (in time and the number of rescuers) was observed using the proposed framework. The suggested framework helps to resolve multidimensional problems in risk management.
\end{abstract}

Keywords: Crisis management; Geographic information system; Round-robin scheduling; Team activities; Time-geography

\section{INTRODUCTION}

Crisis activities like other human activities happen in a specific time and space. Therefore, the success of risk management depends highly on the optimization of time and space where the crisis occurs (Nouri et al., 2010). Numerous researches have been proposed to model crisis activities; but most of them have examined the activities in isolation and put their emphasis on either space or time of a disaster or risk management (Alam et al., 2006; Jaques, 2007; Mohammadi Roozbahani et al., 2009). Any proper planning should then consider both dimensions for modeling and management of crisis activities that are now expanded quickly (Nouri et al., 2008; Vafaeinezhad et al., 2009). There exist several obstacles over widespread utilization of geographic information system (GIS) in crisis management activities (Zerger and Smith, 2003). These include; limited data and software interoperability (Vahidnia et al., 2009), lack of mechanisms for immediate integration of real time geospatial data into the crisis management process (Ahmadian Marj et al., 2009), difficult use of GIS

*Corresponding Author Email: vafaei@alborz.kntu.ac.ir Tel.: +98912 287 5986; Fax: +98218878 6213 interfaces that force crisis managers to rely on GIS technicians to provide necessary information for same -and different- place group work. This research studies crisis activities in multidimensional framework of space and time in GIS environment. Since a team manager, observing his/her group activities in graphic representation (analog modeling) can make better decisions; the proposed framework uses analog presentations. However, in many cases, presenting crisis activities; due to the complex nature of communication; is impossible in analog modeling. Mathematical modeling is more helpful in analyzing such activities. Therefore, the proposed framework enjoys the advantages of analog, as well as mathematical modeling.

Hagerstrand (1970) suggested a framework that became popular as Time-Geography. The framework models human activities in space and time, and lets graphics to be shown on space-time axes (analog modeling). Based on this framework, two right-angled axes can be presented: $\mathrm{X}$ as the space and $\mathrm{Y}$ as the time of activity. Many researchers have been interested in the mentioned framework (Yu and Shaw, 2008), so 
that they applied it in several spatio-temporal human related models, such as: accessibility (Kwan, 1998; Miller, 1999a; Kwan and Weber, 2003; Neutens et al., 2008; Schwanen et al., 2008), human travel (Kwan, 2000; Laube et al., 2007), transport demand modeling (Miller, 1999b; Wang and Cheng, 2001) or have extended the framework in mathematical manner (Miller, 2005). In addition, some other researchers have used the framework in case oriented problems such as deriving rules from activity diary data, measuring similarity between geospatial lifelines (Arentze et al., 2001; Sinha and Mark, 2005). Even though, the framework has the potential to be used in various aspects of human modeling, but dynamic phenomena can hardly be presented on it. To overcome such a shortcoming, this study uses a mathematical model, round-robin scheduling, to extend time-geography for presenting dynamic phenomena. The suggested model can be implemented in GIS environment. Analytical capabilities of GIS are then exercised to manage the risks optimally. To demonstrate the validity of the proposed model, it is applied in the management of simulated life-detecting team and implemented in GIS environment. The study area was located in $36^{\circ} 23^{\prime} 30^{\prime \prime}$ geographical latitude and $59^{\circ} 24^{\prime} 26^{\prime \prime}$ longitud at $5 \mathrm{~km}$ distance of MashhadShandiz road that covers $7 \mathrm{~km}^{2}$. This research has been done in May 2009.

\section{MATERIALS AND METHODS}

An extension of time-geography that considers dynamic phenomena for crisis teams' activities is presented in this section.

\section{Developing time-geography with scheduling}

In spite of its graphical representation, timegeography framework has challenged with dynamic phenomena such as temporal resource allocations. In contrast, scheduling models are used for assigning resources dynamically (Lenzini et al., 2004). Therefore, this research attempts to extend time-geography using scheduling. Hence, a new and dynamic model is presented for modeling activities in space and time.

Scheduling can be divided into two categories: preemptive and non-preemptive (Silberschatz et al., 2004). In preemptive system, when resources are delivered to a process, they cannot be redeemed while the process is done. Design and implementing of such system is simple, but it usually is a time- consuming task. Moreover, since human energy decreases by time, particularly when working in rescue scenarios, such model losses its appropriateness in presenting crisis activities. In non-preemptive, a specific and fixed time (time slice) is determined for every process. The assigned time slice varies based on the type and difficulty of crisis team activities, as well as specific features of the place. This method is called time-sharing (Silberschatz et al., 2004). Therefore, the method can be used as an appropriate technique to model crisis activities.

\section{Criteria for scheduling time-geography}

Before selecting a specific scheduling technique for developing time-geography, the effective factors must be defined as follows:

Fairness: All locations and points should receive equal and fair amount of resources.

Utilization-efficiency: All capabilities of the people should be considered.

Response time: The time needed to respond to activities should be minimized as much as possible.

Turn around: The time duration for doing activities in a particular place should be estimated from the beginning up to the end.

Priorities: Priorities should be determined automatically by the system or external resources as static or dynamic. Static priority does not change in time, thus, it is easier to handle. On the other hand, a dynamic priority changes due to environmental variations; for instance, a priority with a lower level may move into a higher one (Tanenbaum, 2001). Therefore, the scheduling algorithm for developing time-geography framework should apply nonpreemptive method. Furthermore, it should improve the interaction between resources and processes, decrease expenses and consider fairness. It should also consider dynamic priorities and decrease system load.

\section{Round-robin}

Round-Robin scheduling model can appropriately address the above issues and as such, it was selected to extend time-geography. This model is a nonpreemptive one, which would use a cross sectional technique for allocating time to all parts (Lenzini et al., 2004; Rasmussen and Trick, 2008). Determining time slice varies based on locations. For instance, in places where more important activities should be done quickly, the time allocation should be shorter than other areas. 
In this model, all processes are situated in a circling queue. If an activity does not finish in time, the allocated resource will be given to the next process. In other words, when the time slice finishes for a process or an activity, a new and fresh helper is replaced which increases efficiency (Fig. 1).

As Fig. 1 presents, all people that run an activity are kept in a circular queue $\left(\mathrm{P}_{1}\right.$ to $\left.\mathrm{P}_{\mathrm{n}}\right)$. In addition, a time interval is assigned to each person, called its time slice, which is allowed to run. The algorithm starts at the beginning of the list of person descriptor blocks (PDBs), giving each person in turn a chance when time slices become available. Since the system must have a reference to start the list and a reference to the current person, it can easily determine who runs next activity in the destruction area; it just follows the array or chain of PDBs. If the person is still running at the end of the time slice, the system is preempted and gives the task to another person. If the person blocks or finishes before elapsing the time slice, the person is released from the system and added to the end of the queue. Therefore, integrating time-geography and scheduling can solve multidimensional problems in appropriate spaces and lead into results that are more real. The proposed model can be implemented in GIS due to its capabilities in data management and charts representing (Solaimani et al., 2005; Alesheikh et al., 2008; Zerrouqi et al., 2008; Banerjee et al., 2009; Tabesh et al., 2010). In the following, the suggested model is assessed on the activities of an earthquake life-detection team.

\section{Assessing the suggested model}

Because of many countries suffer from earthquake and the importance of rescue, this research modeled and assessed the activities of life-detecting team.

\section{Improving rescue teams performance in space-time}

To model the activities, a map of disaster area is generated as a preparatory step in rescue team operations. In this map, the area is divided into new, ordinary and old building structures. Therefore, it is possible to divide the area into high, medium and low destruction areas after the earthquake. Having determined the area for operation and the magnitude

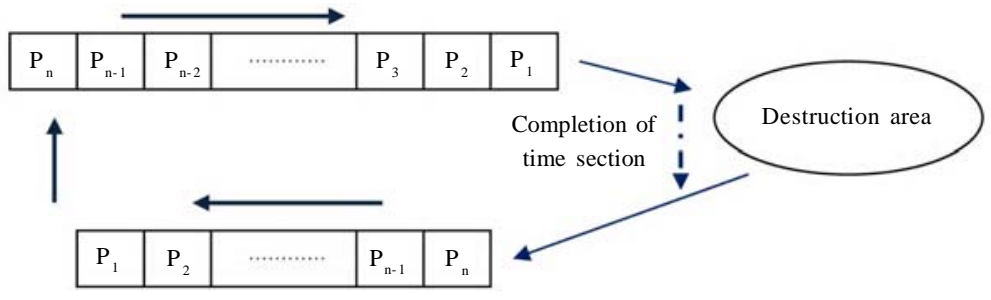

Fig. 1: Use of round-robin scheduling for extending time-geography

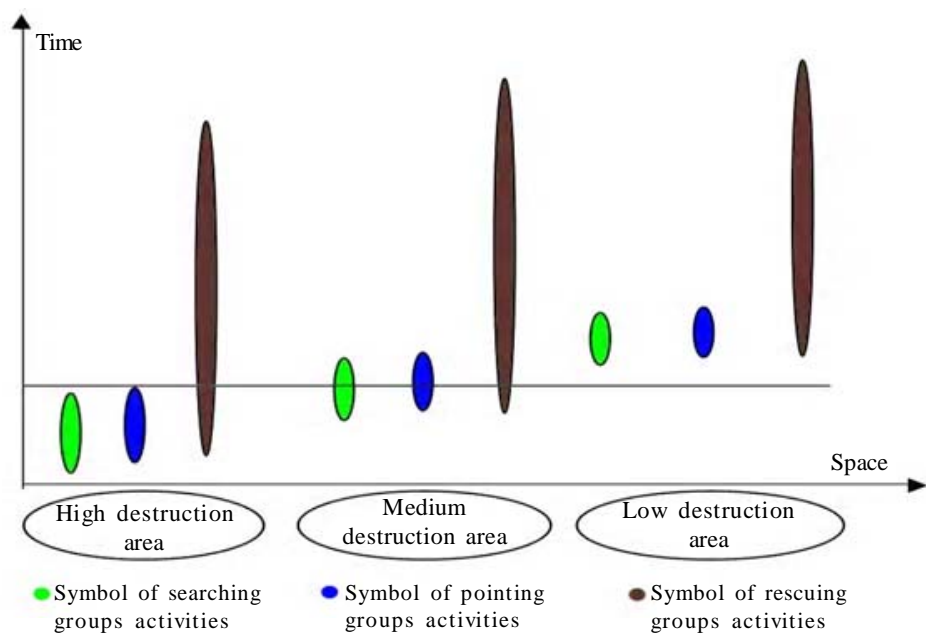

Fig. 2: Modeling of life-detecting activities in a way that their priorities can be set in all area 
of destructions, the team activities; due to their importance and flexibility; should be examined and then shown on space-time axes in time-geography framework. Here, activities of life-detection team that can be divided into searching live people, pointing and rescuing people left under collapses (Schwab, 2003) are presented on space-time axis in a way that their priorities can be set (Fig. 2). In this model, activities are shown separately and the divisions on time axis are relative which entail the priority of each activity. In this model, each activity can have a number, as an identifier and an index as time priority, in order to code the model (from graphic form into pseudo code). In the given model, team arrangement is the task of a team moderator, which is quite familiar with groups' competence and capabilities. Using such a model, team works are facilitated not only on arranging members' activities in space and time, but also on transferring members within groups optimally. For instance, rescuing activities is a very cumbersome task, which requires a lot of energy. In this case, team director using the model and by drawing a line where meeting time axis in the desired time, can easily replace some members from other groups. The director can also arrange members in other low-energy activities shown above the line. This task can in turn increase team efficiency and rescue management. The horizontal line in the above figure indicates that if a member finishes his/her task, he/she can participate in other activities shown above the line depending on their physical status such as remaining energy, type of profession, training experience and the needs in the given area. Using the proposed model, higher efficiency can only be achieved when the action plan is predetermined and unexpected incidents are ignored. Moreover, team members are fixed and no one else can be added to teams. Although such configuration is feasible, it seems very restrictive in many real situations such as rescue works. The main reason is that the groups' members are not predetermined and as time passes, more members are added. If such assumptions are made, perturbation are inevitable. Round-Robin model can easily handle the situation by allocating a time variable to each member. The time variable should be varied based on the type of activities and the energy needed. Here, a fresh person replaces another as soon as his/her activity is accomplished and then the graphical model will change automatically. To increase adaptability, it is possible to use time-geography framework, as well as indexing each activity on spacetime axes.

\section{Practical assessment}

In order to assess the proposed framework, a simulation - a real earthquake was not happened during the research period - is designed and executed. The study area is located in $36^{\circ} 23^{\prime} 30^{\prime}$ ' geographical latitude and $59^{\circ} 24^{\prime} 26^{\prime}$ ' longitudinal in $5 \mathrm{~km}$ distance of Mashhad-Shandiz road that covers $7 \mathrm{~km}^{2}$. The area was divided into three classes: high, medium and low destruction locations. The area is delineated based on the assumed intensity of destruction. The participants are gathered in life-detecting team and their activities were thoroughly assessed. In such simulation, the lifedetecting team includes two searchers, two pointers and four savers. In this rescue operation, as it was impossible to have real persons as victims, it was decided to have nine pets. Of these pets, five were placed in high destruction area, three in medium and one was put in low destruction area. This was performed in a way that no harm could threaten the pets and no one in life-detecting team knows where they are. In the first test, operation was accomplished in a normal way without considering the proposed model. Twenty four team members simultaneously began the rescue operation in the study areas. All activities were recorded including the type of activity, number of members needed, the beginning and ending time, the amount of time needed, the operation site and the distance every member took for each activity. In the second phase, the activities and arrangements were accomplished based on the suggested model. In this phase, the operations were started with eight persons and as time passes, four fresher ones were added to the teams. This is done to test the dynamic nature of the model. The data in the whole operation was accurately recorded to make a precise comparison possible (Tables 1 and 2).

The implementation of the framework was done in ArcGIS software environment to present the capability of the suggested model (Fig. 3).

In this simulation, software customization was achieved by ArcObject programming language. membership functions were defined to change the data of each class according to the model. In addition, loading operator was defined for transferring data to file. Life-detecting super class with searching, pointing and rescuing classes were also developed. The data of Tables 1 and 2 were loaded and the comparison based on the following two cases was made. 
Int. J. Environ. Sci. Tech., 7 (2), 243-250, Spring 2010

Table 1: Life-detecting operation normally

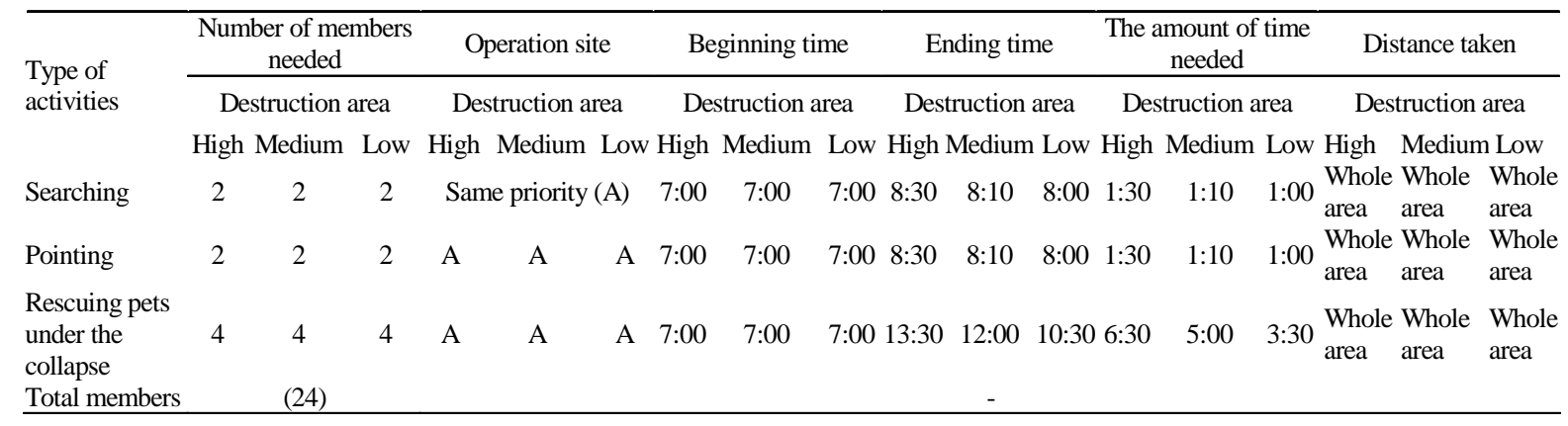

Table 2: Life-detecting operation using suggested model

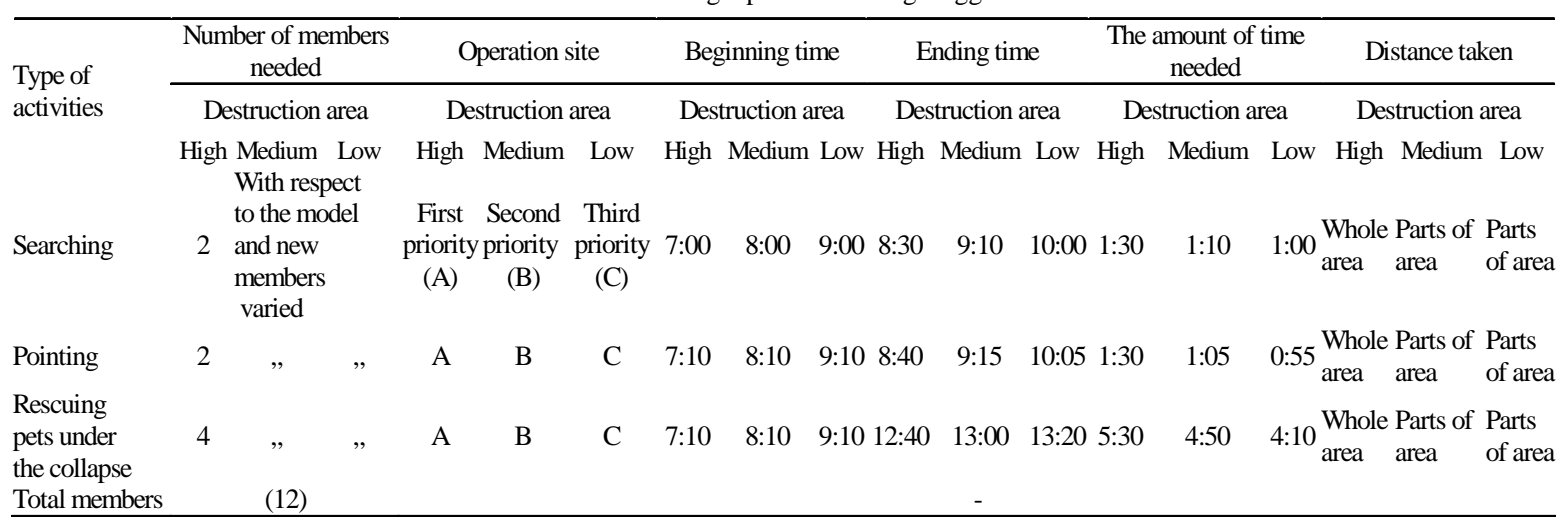

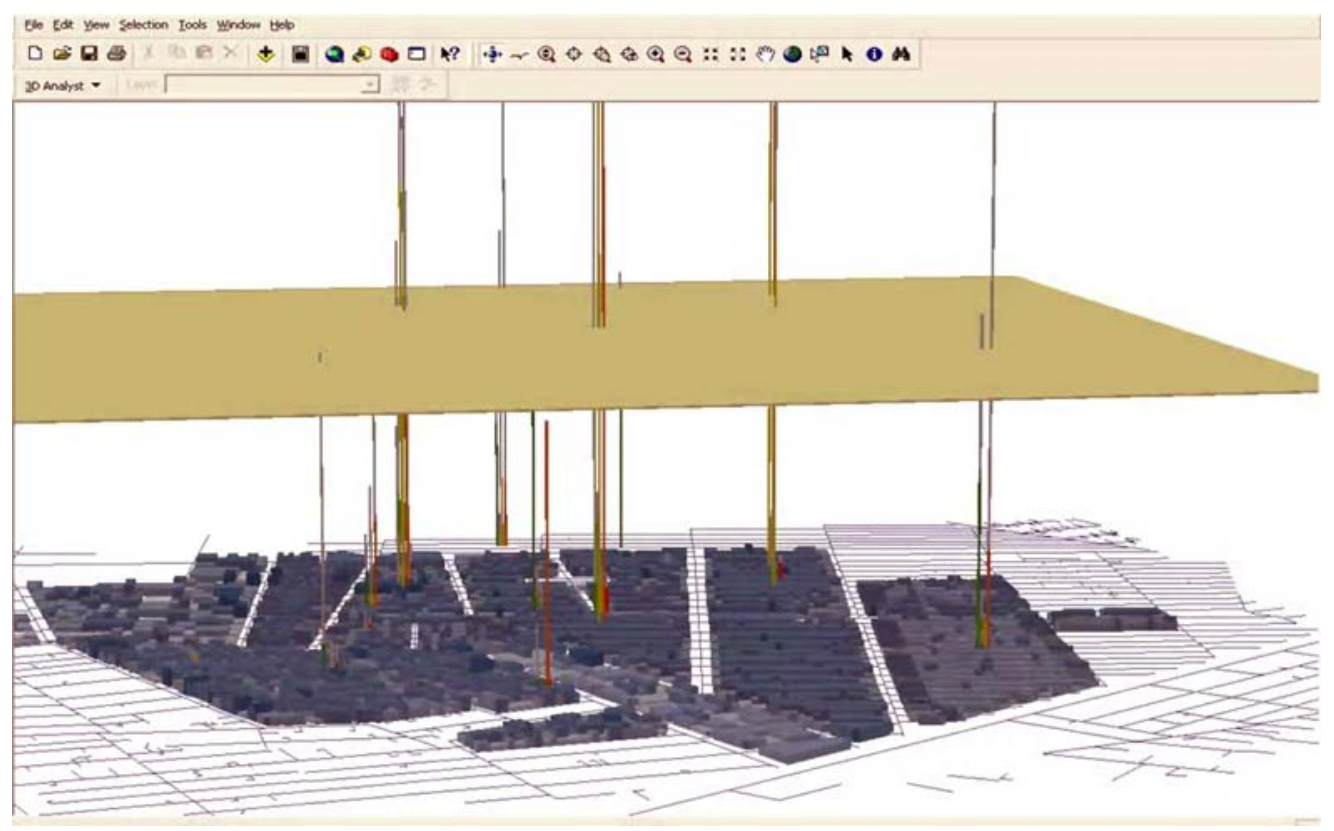

Fig. 3: Implementation of the suggested model in ArcGIS environment to present the capability of the suggested model 
A. R. Vafaeinezhad et al.

Table 3: The results of the first test

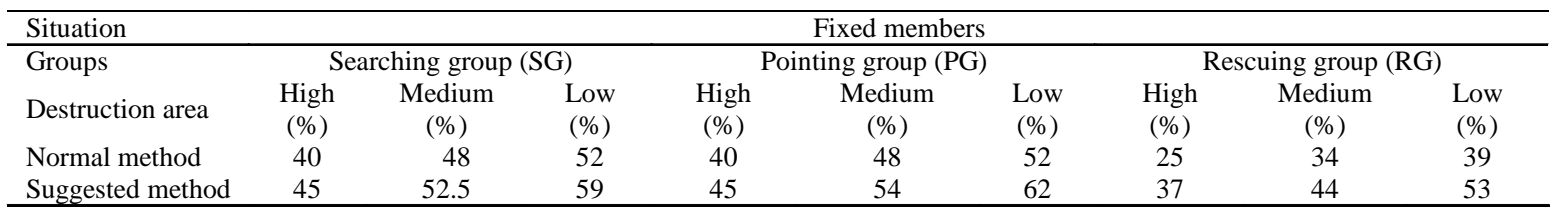

Table 4: The result of the second test

\begin{tabular}{|c|c|c|c|c|c|c|c|c|c|}
\hline \multirow{3}{*}{$\begin{array}{l}\text { Situation } \\
\text { Groups } \\
\text { Destruction area }\end{array}$} & \multicolumn{9}{|c|}{ Changing members } \\
\hline & \multicolumn{3}{|c|}{ Searching group (SG) } & \multicolumn{3}{|c|}{ Pointing group (PG) } & \multicolumn{3}{|c|}{ Rescuing group (RG) } \\
\hline & $\begin{array}{l}\text { High } \\
(\%)\end{array}$ & $\begin{array}{l}\text { Medium } \\
\text { (\%) }\end{array}$ & $\begin{array}{l}\text { Low } \\
(\%)\end{array}$ & $\begin{array}{l}\text { High } \\
(\%)\end{array}$ & $\begin{array}{l}\text { Medium } \\
\text { (\%) }\end{array}$ & $\begin{array}{l}\text { Low } \\
\text { (\%) }\end{array}$ & $\begin{array}{l}\text { High } \\
(\%)\end{array}$ & $\begin{array}{l}\text { Medium } \\
\text { (\%) }\end{array}$ & $\begin{array}{l}\text { Low } \\
\text { (\%) }\end{array}$ \\
\hline Suggested method & 53 & 59 & 65 & 84 & 93 & 97 & 55 & 63 & 70 \\
\hline
\end{tabular}

Table 5: Final result determination

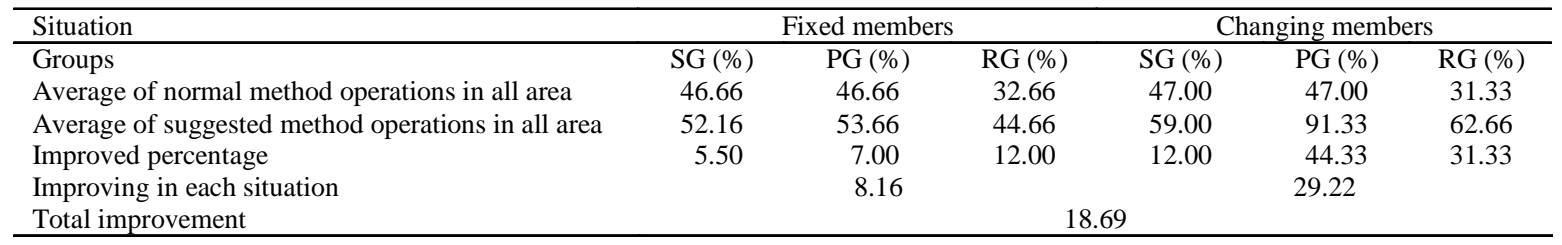

Case 1: Each team started its activity by fixed number of members. Due to the tiredness and work condition, the members operation and their output decreased after a while. Table 3 clearly presents the results of a fixed time rescuing. It also presents the comparison between the normal procedure and the suggested fixed -time method presented in this study. In a normal working procedure, rescuers start their works without any predefined plan and in a perturbation manner. The normal method demonstrated confusion that leads to mismanagement. The table clearly presents the effectiveness of the proposed method. The value of each cell is a function of time and persons needed for accomplishing a task.

Case 2: In this case, the number of members can change and the manager can add up fresh members, hence flexibility is achieved. Table 4 presents the results of this test. It demonstrates the efficiency of the proposed framework.

Considering the assessments, it is clear that the suggested model improves performance in all situations that ranges from $8.16 \%$, to $29.22 \%$. The average improvement is calculated as $18.69 \%$, Table 5 reveals the analogy of teams operations on the zone.

\section{RESULTS AND DISCUSSION}

The occurrence of earthquake threatens many countries in the world. Man has no role in this occurrence but his integrated treatment can reduce suffering and improve the condition. The challenge, however, is to consider time and space simultaneously in modeling rescue operation. Various studies have been performed to manage rescue works but most of them put their emphasis on either space or time of a disaster. For solving the challenges, in the present study, rescue activities in life-detecting teams were modeled in a new and spatio-temporal framework with the aid of round-robin scheduling, time-geography and GIS capabilities. Two scenarios were planned to assess the results of the suggested model. They were; fixed members with no time constraint and variable members with no time limitation. The statistical analyses of the results indicated that the efficiency can be increased applying the suggested model.

\section{CONCLUSION}

Crisis activities usually occur in space and time. Therefore, to manage the human activities and interactions optimally, two aspects of space and time should be considered simultaneously. This study 
presents a new, multidimensional and dynamic framework to increase teamwork efficiency by spatiotemporal modeling of human group activities in crisis management. To do so, human group activities are modeled based on the vertical bars of time-geography framework in space and time. A round-robin scheduling is then used to extend time-geography to manage human activities in a dynamic framework that can be implemented in GIS environment. The suggested model was assessed based on a simulated life-detecting scenario. Software is then developed through ArcObject programming in ArcGIS environment. The statistical abstract of the results showed that the suggested model increased the efficiency up to 18.69 $\%$ in average contrasting with normal methods used by current rescue teams. Future researchers can develop the model through space-time prism and region connection calculus (RCC), which adds the prediction and mathematical human relations to the model.

\section{REFERENCES}

Ahmadian Marj A.; Mobasheri, M. R.; Valadanzouje M. J.; Rezaei Y.; Abaei M. R., (2009). Using satellite images in determination of malaria outbreaks potential region. Environ. Hazard., 8 (2), 89-100 (12 pages).

Alam, Md. J. B.; Alam, M. J. B.; Rahman, M. H.; Khan, S. K.; Munna, G. M., (2006). Unplanned urbanization: Assessment through calculation of environmental degradation index. Int. J. Environ. Sci. Tech., 3 (2), 119-130 (12 pages).

Alesheikh, A. A.; Soltani, M. J.; Nouri, N.; Khalilzadeh, M., (2008). Land assessment for flood spreading site selection using geospatial information system. Int. J. Environ. Sci. Tech., 5 (4), 455-462 (8 pages).

Arentze, T.; Hofman, F.; Timmermans, H., (2001). Deriving rules from activity diary data: A learning algorithm and results of computer experiments. J. Geogr. Syst., 3 (4), 325346 (22 pages).

Banerjee, D.; Chakraborty, S. K.; Bhattacharyya, S.; Gangopadhyay, A., (2009). Appraisal and mapping the spatial-temporal distribution of urban road traffic noise. Int. J. Environ. Sci. Tech., 6 (2), 325-335 (11 pages).

Hagerstrand, T., (1970). What about people in regional science. Region. Sci. Assoc., 24 (1), 7-24 (18 pages).

Jaques, T., (2007). Issue management and crisis management: An integrated, non-linear, relational construct. Pub. Relat. Rev., 33 (2), 147-157 (11 pages).

Kwan, M., (1998). Space-time and integral measures of individual accessibility: A comparative analysis using a pointbased network. Geogr. Anal., 30 (3), 191-216 (26 pages).

Kwan, M., (2000). Interactive geovisualization of activitytravel patterns using three-dimensional geographical information systems: A methodological exploration with a large data set. Trans. Res. Part. C: Emerg Technol., 8 (1), 185-203 (10 pages).

Kwan, M.; Weber, J., (2003). Individual accessibility revisited: implications for geographical analysis in the twenty-first century. Geogr. Anal., 35 (4), 341-353 (13 pages).

Laube, P.; Dennis, T.; Forer, P.; Walker, M., (2007). Movement beyond the snapshot-Dynamic analysis of geospatial lifelines. Comput. Environ. Urban Syst., 31 (5), 481-501 (21 pages).

Lenzini, L.; Mingozzi, E.; Stea, G., (2004). Eligibilityb-Based round robin for fair and efficient packet scheduling in interconnection networks. IEEE Trans. Parallel Distrib. Syst., 15 (3), 244-256 (13 pages).

Miller, H., (2005). A measurement theory for time geography. Geogr Anal., 37 (1), 17-45 (29 pages).

Miller, H. J., (1999a). Measuring space-time accessibility benefits within transportation networks: Basic theory and computational procedures. Geogr. Anal., 31 (2), 187-212 (26 pages).

Miller, H. J., (1999b). Potential contributions of spatial analysis to geographic information systems for transportation. Geogr. Anal., 31 (4), 373-399 (27 pages).

Mohammadi Roozbahani, M.; Nassiri, P.; Jafari Shalkouhi, P., (2009). Risk assessment of workers exposed to noise pollution in a textile plant. Int. J. Environ. Sci. Tech., 6 (4), 591-596 (6 pages).

Neutens, T.; Van de Weghe, N.; Witlox, F.; De Maeyer, P., (2008). A three-dimensional network-based space-time prism. J. Geogr. Syst., 10 (1), 89-107 (19 pages).

Nouri, J.; Karbassi, A. R.; Mirkia, S., (2008). Environmental management of coastal regions in the Caspian Sea. Int. J. Environ. Sci. Tech., 5 (1), $43-52$ (10 pages).

Nouri, J.; Omidvari, M.; Tehrani, S. M., (2010). Risk assessment and crisis management in gas stations. Int. J. Environ. Res., 4 (1), 143-152 (10 pages).

Rasmussen, R. V.; Trick, M. A., (2008). Round robin scheduling - a survey. Eur. J. Oper. Res., 188 (3), 617-636 (20 pages).

Schwab, J., (2003). Planning for post-disaster recovery and reconstruction. Federal Emergency Management Agency, USA.

Schwanen, T.; Kwan, M.; Ren, F., (2008). How fixed is fixed? Gendered rigidity of space-time constraints and geographies of everyday activities. J. Geoforum., 39 (6), 2109-2121 (13 pages).

Silberschatz, A.; Galvin, P.; Gagne, G., (2004). Operating system concepts with Java, $6^{\text {th. }}$ Ed. John Wiley and Sons, 52-67.

Sinha, G.; Mark, D., (2005). Measuring similarity between geospatial lifelines in studies of environmental health. J. Geogr. Syst., 7 (1), 115-136 (22 pages).

Solaimani, K.; Mohammadi, H.; Ahmadi, M. Z.; Habibnejad, M., (2005). Flood occurrence hazard forecasting based on geographical information system. Int. J. Environ. Sci. Tech., 2 (3), 253-258 (6 pages).

Tabesh, M.; Delavar, M. R.; Delkhah, A., (2010). Use of geospatial information system based tool for renovation and rehabilitation of water distribution systems. Int. J. Environ. Sci. Tech., 7 (1), 47-58 (12 pages).

Tanenbaum, A. S., (2001). Modern Operating Systems, $2^{\text {nd. }}$ Ed. Prentice Hall, 32-43.

Vafaeinezhad, A. R.; Alesheikh, A. A.; Hamrah, M.; Nourjou, R.; Shad, R., (2009). Using GIS to develop an efficient spatio-temporal task allocation algorithm to human groups in an entirely dynamic environment. Case study: Earthquake rescue teams. Springer-Verlag Berlin Heidelberg, LNCS $5592,66-78$. 
Vahidnia, M. H.; Alesheikh, A. A.; Alimohammadi, A., (2009). Hospital site selection using fuzzy AHP and its derivatives. J. Environ. Manage., 90 (10), 3048-3056 (9 pages).

Wang, D.; Cheng, T., (2001). A Spatio-temporal data model for activity-based transport demand modelling., Int. J. Geogr. Inform. Sci., 15 (6), 561-585 (25 pages).

Yu, H.; Shaw, S. L., (2008). Exploring potential human activities in physical and virtual spaces: A spatio-temporal GIS approach. Int. J. Geogr. Inform. Sci., 22 (4), 409-430 (22 pages).
Zerger, A.; Smith, D. I., (2003). Impediments to using GIS for real-time disaster decision support. Comput. Environ. Urban Syst., 27 (2) 123-141 (19 pages).

Zerrouqi, Z.; Sbaa, M.; Oujidi, M.; Elkharmouz, M.; Bengamra, S.; Zerrouqi, A., (2008). Assessment of cement's dust impact on the soil using principal component analysis and GIS. Int. J. Environ. Sci. Tech., 5 (1), 125-134 (10 pages).

AUTHOR (S) BIOSKETCHES

Vafaeinezhad, A. R., Ph.D. Candidate, Department of GIS, Faculty of Geomatics Engineering, K.N. Toosi University of Technology, Tehran, Iran. Email:vafaei@alborz.kntu.ac.ir

Alesheikh, A. A., Ph.D., Associate Professor, Department of GIS, Faculty of Geomatics Engineering, K.N. Toosi University of Technology, Tehran, Iran. Email: alesheikh@kntu.ac.ir

Nouri, J., Ph.D., Full Professor, Department of Environmental Management, Graduate School of the Environment and Energy, Science and Research Branch, Islamic Azad University, Tehran, Iran. Email: nourijafar@gmail.com

How to cite this article: (Harvard style)

Vafaeinezhad, A. R.; Alesheikh, A. A.; Nouri, J., (2010). Developing a spatio-temporal model of risk management for earthquake life detection rescue team. Int. J. Environ. Sci. Tech., 7 (2), 243-250. 